March 1996

\title{
Radiative Seesaw Mechanism at Weak Scale
}

\author{
Zhijian Tao \\ Theory Division, Institute of High Energy Physics, Academia Sinica \\ Beijing 100039, China
}

\begin{abstract}
We investigate an alternative seesaw mechanism for neutrino mass generation. Neutrino mass is generated at loop level but the basic concept of usual seesaw mechanism is kept. One simple model is constructed to show how this mechanism is realized. The applications of this seesaw mechanism at weak scale to cosmology and neutrino physics are discussed.
\end{abstract}


Seesaw mechanism [1] is one of the best and simplest ways to understand why neutrino, if massive, is much lighter than the corresponding charged lepton in the same generation. The central idea of the seesaw is to introduce a right-handed neutrino $\nu_{R}$, which will couple to lepton doublet through Yukawa coupling. The point is that besides the Yukawa interaction term there is another bare Majorana mass term $M_{R}$ for $\nu_{R}$. After the gauge symmetry breaking the Yukawa term will result in a Dirac neutrino mass $m_{D}$. Therefore the neutrino mass matrix takes the form

$$
\left(\begin{array}{cc}
0 & m_{D} \\
m_{D}^{+} & M_{R}
\end{array}\right)
$$

In the three generation model $m_{D}$ and $M_{R}$ are three by three mass matrix. Diagonalizing the mass matrix one gets the neutrino mass eigenstates. If $M_{R}$ is much bigger than $m_{D}$ the mass of the light neutrinos, which are mostly left-handed, is determined as $m_{D}^{T} M_{R}^{-1} m_{D}$. The heavy states, which are mostly right-handed, have mass almost as $M_{R}$. Therefore one sees that even if the Dirac mass term is comparable to the charged lepton mass the light neutrino mass can be much smaller. The features one should notice in this mechanism are the following: $M_{R}$ is a free scale usually taken from weak scale to the Grand Unification Scale (GUT). And the heavy neutrinos are not stable, they decay through mixing to the light neutrinos. For large $M_{R}$ the heavy neutrinos decay very fast, so they have no cosmological consequence. In this mechanism the lepton number symmetry is broken either explicitly or spontaneously. Although smallness of the neutrino mass can be understood in this mechanism, the actual values of neutrino mass and mixing are not predicted due to the unknown scale $M_{R}$ and structure of $m_{D}$. As an indication, if one assumes that $m_{D}$ is same as the charged lepton mass matrix and $M_{R}$ is a unit matrix up to a scale, one gets the relations for the light neutrino masses $m_{\nu_{i}}=m_{i}^{2} / M_{R}$, where the index i denotes the i-th generation. So it is the scale $M_{R}$ determines the order of the magnitude of the neutrino mass. If $M_{R}$ is at GUT scale, one obtains $m_{\nu_{e}}<<m_{\nu_{\mu}}<<m_{\nu_{\tau}} \leq 10^{-3} \mathrm{eV}$. These tiny masses may only play a role 
for solar neutrino behavior. Another most interesting scale is the weak scale. There are a number of physical motivation to consider $M_{R}$ at weak scale. First of all for weak scale $M_{R}$ the new physics mechanism can be tested in the future experiments, secondly it avoids to introduce an intermediate scale between weak and GUT scales. For $M_{R}$ at weak scale all three light neutrino masses are close to their upper bound, i.e. a few eV, $100 \mathrm{KeV}$ and $10 \mathrm{MeV}$ for electron, muon and tau neutrinos. These neutrinos are strongly constrained from cosmological and astrophysical consideration depending on their decay modes [2]. Obviously they offer no solutions to the solar neutrino and atmospheric neutrino problems [3], but they may play a role in the dark matter issue by providing either a hot dark matter component in the mixed dark matter model [4] or a late decaying particle [5, 6] in the cold dark matter model [7]. And no cold dark matter candidate is provided. Moreover there may be a problem for the seesaw model in consideration of the baryogenesis of the universe. The problem is due to $\mathrm{B}-\mathrm{L}$ (baryon number minus lepton number) symmetry violation. Once the $\mathrm{B}-\mathrm{L}$ violation process through seesaw mechanism and the anomalous $\mathrm{B}+\mathrm{L}$ process induced by gauge interaction are in the thermal equilibrium at an early stage of the universe [8], any primordial origin of baryon and lepton asymmetries generated earlier are washed out. It leads to very strong constrains on the neutrino mass [9]. An upper bound of a few eV for all three light neutrino masses are obtained in order to avoid this problem [10], which in turn implies the scale of $M_{R}$ should be much larger than weak scale. However, this problem can be evaded if the B-L symmetry is spontaneously broken at the weak scale. Before B-L symmetry breaking only $\mathrm{B}+\mathrm{L}$ violating process due to gauge anomaly is active and after the B-L symmetry breaking the anomalous $\mathrm{B}+\mathrm{L}$ violating process is already suppressed as the temperature of universe is low enough. These two processes will never be in thermal equilibrium through the evolution of the universe. Hence the constrains on the strength of B-L violation from the baryogenesis of the universe is avoided [11]. 
In this letter we consider a different scheme for seesaw mechanism. The main consideration is to keep the basic concept of the seesaw mechanism, i.e. the light neutrino mass is suppressed by the large right-handed neutrino mass $M_{R}$, and require the neutrino mass only generated radiatively [12]. For this kind of scenario neutrino mass is expressed as $m_{\nu} \sim \frac{\lambda}{16 \pi^{2}} m_{D}^{T} M_{R}^{-1} m_{D}$. One sees that adding to the usual seesaw form is another suppression factor from loop effect. $\lambda$ is some combination of the coupling constants besides Yukawa coupling. This constant can be very small naturally if it is associated with the lepton number violation. Therefore the neutrino mass is at least two order of magnitude smaller than that in the usual seesaw model for the same scale $M_{R}$. This scenario has some very interesting features. First of all $\nu_{R}$ can be stable by imposing some discrete symmetries, while still giving light neutrino nonzero mass. In fact, in order to avoid tree-level Dirac neutrino mass these symmetries are necessary. This is very different from the original seesaw mechanism, where $\nu_{R}$ is unstable for nonzero light neutrino mass. The application of the stable $\nu_{R}$ is to play a role of the cold dark matter. Secondly light neutrino mass is suppressed also by the loop effect, so for a weak scale $M_{R}$ the neutrino mass can be much smaller than the current experimental bounds. The light neutrino may be provided as a candidate for explaining the solar neutrino, atmospheric neutrino problems and a hot dark matter component in the mixed dark matter model or still a late decaying particle in the cold dark matter model. Baryogenesis of the universe also restricts this kind of mechanism, but the constrains are relaxed due to the loop factor. The most attractive picture is that if there is no other scale except weak scale below GUT, $M_{R}$ is around this scale, then in this scenario with only one scale and with only neutrino particles, one may explain the observed dark matter problem and the structure formation of the universe, and possibly other related phenomena in neutrino physics. From now on we will call the original seesaw mechanism as tree-level seesaw mechanism and the other radiative seesaw mechanism. 
Now let us implement this mechanism in a very simple model. This model is to extend the standard model by introducing three family of right-handed neutrinos $\nu_{R}$ and one more Higgs doublet $\Phi$. We impose an $Z_{2}$ discrete symmetry on this model. Under this symmetry transformation $\nu_{R}$ and $\Phi$ change sign, while other fields remain same. As a result of this symmetry, $\nu_{R}$ does not couple to the standard Higgs $\Phi_{S}$ through Yukawa coupling. Only the new Higgs doublet $\Phi$ couples to $\nu_{R}$. Now we can write down all the possible interaction terms for this model. It includes the gauge interaction, Yukawa coupling and the Higgs potential. However in this work only the Yukawa interaction for lepton and part of the Higgs potential are relevant. The Yukawa coupling is expressed as following:

$$
L_{Y}=f_{i j} \bar{l}_{i} e_{R j} \Phi_{S}+g_{i j} \bar{l}_{i} \nu_{R j} \Phi+h . c+M_{i j} \nu_{R i}^{T} \nu_{R j}
$$

Here $l_{i}$ and $e_{R i}$ are lepton doublet and the right-handed charged lepton respectively. Since the $Z_{2}$ symmetry is exact and will not be broken, $\Phi$ will not develop a nonzero vacuum expectation value $(\mathrm{VEV})$. Therefore with only $L_{Y}$ lepton number is not broken, i.e. neutrino does not obtain mass at this level. However with all the terms in $L_{Y}$ and a term like $\lambda\left(\Phi{ }_{S}^{+} \Phi\right)^{2}$ in the potential, it is easy to check that lepton number symmetry is not automatically conserved anymore. In other words neutrino must develop a nonzero mass, but obviously this mass is generated only at loop level. If the masses of $\Phi$ and $\nu_{R}$ are at the same order of the magnitude $M_{R}$, the light neutrino mass can be estimated as, up to a logarithmic factor,

$$
m_{\nu} \simeq \frac{\lambda}{16 \pi^{2}} g^{T} M_{R}^{-1} g V^{2}
$$

where $V$ is the VEV of the standard Higgs $\Phi_{S}$. In the tree-level seesaw mechanism it is assumed that the couplings $g$ and $f$ have same order of magnitude and similar structure, that is $g V \sim f V=m_{D}$. Then in this model the basic seesaw concept is realized at the loop level. Compared with the simplest tree level seesaw model, which is the standard model plus right-handed neutrino, our model only has one more Higgs doublet and introduce an 
additional $Z_{2}$ discrete symmetry. Because the $Z_{2}$ symmetry is not broken, $\Phi_{S}$ and $\Phi, \nu_{R}$ and $\nu_{l}$ will not mix with each other respectively. The lightest particles among $\nu_{R}$ and $\Phi$ are stable. In above description the lepton number is explicitly broken. Of course the lepton number may also be broken spontaneously with introduction of some singlet scalar fields as in the singlet majoron model [13]. In the singlet majoron model the mass term of $\nu_{R}$ is replaced by $h \nu_{R} \nu_{R} S$, where $S$ is the singlet scalar field. When $S$ field gets a nonzero VEV, lepton number is spontaneously broken. The difference between tree-level seesaw model and our model is that in our model the majoron only couples to $\nu_{R}$ at tree level. The light neutrino couples to majoron not through mixing but radiative correction.

Now we come to discuss the application of our model to the dark matter issue of the universe and other issues in neutrino physics. The very interesting question is to see how the right-handed neutrino may serve as the candidate of cold dark matter. In our model in principle both $\nu_{R}$ and $\Phi$ can be the candidate of the cold dark matter depending on which particle is the lightest one. Here we assume that one of $\nu_{R}$ is the lightest particle among $\nu_{R}$ and $\Phi$, and from now on we just call it $\nu_{R}$. Because $\Phi$ has direct standard gauge coupling to $\mathrm{Z}$ boson, if it is the dark matter the elastic scattering cross section of $\Phi$ from the nuclei of the detector is determined by this neutral current interaction. Having not observed any signal of this reaction requires the mass of $\Phi$ to be at least a few $\mathrm{TeV}$ [14. On the other hand $\nu_{R}$ dark matter is not constrained much from the direct dark matter search experiments. The relic abundance of $\nu_{R}$ is controlled by its interaction with other light particles and the evolution of the universe. We are going to estimate the relic density of $\nu_{R}$ in our model with and without majoron.

First let us see the case without majoron. Most generally the evolution of $\nu_{R}$ is determined by the combined evolution equations of $\nu_{R}$ and $\Phi$. The equations include the contributions from $\bar{\nu}_{R} \nu_{R}$ annihilation, $\Phi \Phi$ annihilation and the decay from $\Phi$ to $\nu_{R}$. If $\Phi$ 
is not almost degenerate with $\nu_{R}$, i.e. the mass difference $\Delta M$ is significantly larger than the freezeout temperature of $\nu_{R}$, one may neglect the presence of $\Phi$. Then only the $\bar{\nu}_{R} \nu_{R}$ annihilation cross section $\left\langle\sigma_{A} v>\right.$ determines the relic density of $\nu_{R}$. Approximately the contribution of the thermal relics of a massive cold dark matter particle to mass density of the universe can be expressed as $\Omega h^{2} \sim 10^{-37} \mathrm{~cm}^{2} /<\sigma_{A} v>$ [15]. To be the candidate of the cold dark matter, its annihilation cross section should be roughly as large as $10^{-37} \mathrm{~cm}^{2}$. And the freezeout temperature $T_{D}\left(\nu_{R}\right)$ at which $\nu_{R}$ decouples from the thermal equilibrium is about $M_{R} / 20$. For $\bar{\nu}_{R} \nu_{R}$ annihilation the dominant channel is $\bar{\nu}_{R} \nu_{R} \rightarrow \bar{\nu}_{l} \nu_{l}, \quad e^{+} e^{-} \ldots$, which is related to the light neutrino mass generation. The cross section for this channel is estimated as

$$
<\sigma_{A} v>\sim \frac{\lambda^{\prime 2} m_{D}^{4}}{\pi M_{R}^{6}}=10^{-39}\left(\frac{\lambda^{\prime}}{1.0}\right)^{2}\left(\frac{m_{D}}{1.7 G e V}\right)^{4}\left(\frac{100 G e V}{M_{R}}\right)^{6} \mathrm{~cm}^{2}
$$

where $\lambda^{\prime}$ represents all possible contribution from the scalar potential including $\lambda$ term. Since $\lambda$ is related to the lepton number violation, $\lambda^{\prime}$ can be naturally much larger than $\lambda$. In fact with the parameters chosen reasonably as in above equation, the annihilation cross section is much smaller than what needed for $\nu_{R}$ being the cold dark matter, $\nu_{R}$ contribution overcloses the universe. On the other hand, however, if $\Delta M$ is much smaller than $T_{D}\left(\nu_{R}\right)$, the density of $\Phi$ and $\nu_{R}$ are both determined by annihilation process $\Phi \Phi \rightarrow$ light standard model particles. The cross section is estimated as

$$
<\sigma v>\simeq \frac{\pi \alpha^{2}}{M_{R}^{2}} \simeq 10^{-35}\left(\frac{100 G e V}{M_{R}}\right)^{2} \mathrm{~cm}^{2}
$$

where $\alpha$ is the fine structure constant. At this extreme situation with the parameters chosen as in the equation (5), the annihilation process is too strong, it contributes only a small portion of needed dark matter density. Although for $\Delta M$ between these two extreme situation one needs to solve the combined evolution equations, one can certainly expects for a certain range of $\Delta M$ from $T_{D}\left(\nu_{R}\right)$ to $M_{R}$, the relic $\nu_{R}$ is able to contribute a closure 
density to the universe. A similar case is investigated quantitatively but in a different model [16]. Its numerical calculation supports this expectation in our model.

As we already mentioned the light neutrino mass depends on the parameter $\lambda$. With $\lambda \leq 1$, one obtains $m_{\nu_{\tau}} \leq 100 \mathrm{KeV}, m_{\nu_{\mu}} \leq 300 \mathrm{eV}, m_{\nu_{e}} \leq 10^{-2} \mathrm{eV}$. We investigate three possible choices for neutrino mass, which are interesting in neutrino physics. The first is $m_{\nu_{\tau}} \simeq 5 \mathrm{eV}$, then $\nu_{\tau}$ can be the hot dark matter component needed for the large scale structure formation in mixed cold dark matter model. In this case $\nu_{\mu}$ mass is close to $10^{-2} \mathrm{eV}$ and $\nu_{e}$ is very light as expected from seesaw. If the mass of $\nu_{\mu}$ is a few times smaller than $10^{-2} \mathrm{eV}, \nu_{\mu}, \nu_{e}$ oscillation may offer a solution to solar neutrino problem through MSW mechanism. If it is a few times larger, the mass square difference for these two neutrino species are just what needed for atmospheric neutrino problem. Because all three light neutrino masses are very small, the constrain from the baryogenesis of the universe, which requires the primordial baryon asymmetry is not washed out by the coexistence of $\mathrm{B}-\mathrm{L}$ violation process for neutrino mass generation and gauged $\mathrm{B}+\mathrm{L}$ violation process, is satisfied. The second choice is to have the mass of $\nu_{\tau}$ around $0.1 \mathrm{eV}$, and mass of $\nu_{\mu}$ around $3 \times 10^{-3} \mathrm{eV}$ and $\nu_{e}$ much lighter. In this case three neutrino oscillation can possibly explain both solar and atmospheric neutrino problems, but no candidate of hot dark matter is provided. The third choice is with three neutrinos as heavy as about $1 \mathrm{KeV}, 5 \mathrm{eV}$ and $10^{-4} \mathrm{eV}$. With this neutrino masses, $\nu_{\mu}$ may serve as the candidate of hot dark matter and the oscillation between $\nu_{\mu}$ and $\nu_{e}$ can explain the LSND neutrino oscillation experimental data [17]. However the $\mathrm{KeV} \tau$ neutrino must decay fast enough in order not to delay the beginning of matter dominated epoch of the universe too much. This demands the lifetime $\tau\left(\nu_{\tau}\right) \leq 2 \times 10^{2}\left(\frac{1 \mathrm{KeV}}{m_{\nu_{\tau}}}\right)^{2} y r$ [18. In our model the dominant decay modes for $\nu_{\tau}$ are $\nu_{\tau} \rightarrow \nu_{(\mu, e)}+(\mu, e)^{ \pm}$. Its lifetime is therefore estimated as $\tau\left(\nu_{\tau}\right) \geq 10^{12}\left(\frac{\mathrm{KeV}}{m_{\nu_{\tau}}}\right)^{5} \mathrm{yr}$. We see that this constrain along rules out the third choice. 
Now we proceed to discuss the majoron model. In the majoron model the relic density of $\nu_{R}$ is not only determined by the annihilation processes $\bar{\nu}_{R} \nu_{R} \rightarrow \bar{\nu}_{l} \nu_{l}, e^{+} e^{-} \ldots$ but also by the process $\nu_{R} \nu_{R} \rightarrow \phi_{R} \phi_{R}$, here $\phi_{R}$ is the majoron associated with spontaneous lepton number breaking. The coupling between $\phi_{R}$ and other standard model particles is only induced by loop effect and proportional to some power of Yukawa coupling, so it is negligible in considering of the relic density of $\nu_{R}$. We estimate the cross section for the second annihilation process $\nu_{R} \nu_{R} \rightarrow \phi_{R} \phi_{R}$ as

$$
<\sigma_{A} v>\sim \frac{h^{4}}{3 \pi M_{R}^{2}}\left(\frac{P}{E}\right)^{2} \simeq \frac{h^{4} T}{\pi M_{R}^{3}}
$$

in terms of the energy $\mathrm{E}$ and three momentum $\mathrm{P}$ of $\nu_{R}$ in the center of mass frame, and $\mathrm{T}$ is the temperature of the universe. It is noticed that for this process it is the p-wave dominated. The s-wave contribution is forbidden as a result of momentum and CP conservation as well as the statistics. This is roughly a weak interaction cross section if $h$ is around order of one and $M_{R}$ at weak scale. Since the second process dominates over the first annihilation process, it is the second annihilation cross section determines the relic density of $\nu_{R}$ at present. To get a feeling of the numbers, $\left\langle\sigma_{A} v>\sim 10^{-37} \mathrm{~cm}^{2}\right.$ with $h \sim 0.1$ and $M_{R} \sim 100 \mathrm{GeV}$. Since $\phi_{R}$ decouples from the standard model particles at a high energy scale $\sim M_{R} / 20$ larger than a few $\mathrm{GeV}$, majoron contributes to the effective number of light neutrino species $N_{\nu}$ less than 0.1 when primordial nucleosynthesis commences. Hence the condition $N_{\nu} \leq 3.3$ at the time of nucleosynthesis [19 is satisfied. Other restriction from cosmology and astrophysics can also be easily obeyed. The strongest one is due to the cooling of red giants. It requires the coupling between electron pair and majoron is weaker than $10^{-11}$ [20]. In our model this coupling is safely smaller than this number because this coupling is induced through one loop diagram and is proportional to the square of electron mass.

The distinguished feature of the majoron model is that it offers new decay channels for the heavier light neutrino. In our model $\nu_{\tau}$ can decay to other two lighter neutrinos plus a 
majoron. We consider one interesting situation here, mass of $\nu_{\tau}$ is about order of $10 \mathrm{KeV}$, $\nu_{\mu}$ is a few $\mathrm{eV}$. In previous model without majoron this possibility is ruled out. However due to the new decay channel to majoron the life time of $\nu_{\tau}$ can be much shorter. The dominant decay channel is to $\nu_{\mu}$ plus a majoron. We estimate the life time of $\nu_{\tau}$ as

$$
\tau\left(\nu_{\tau}\right) \simeq 16 \pi\left(\frac{m_{\nu_{\tau}}}{M_{R}}\right)^{-4} m_{\nu_{\tau}}^{-1}=10^{3}\left(\frac{10 K e V}{m_{\nu_{\tau}}}\right)^{5}\left(\frac{M_{R}}{100 G e V}\right)^{4} y r
$$

Its dependence on the light neutrino mass is similar as that in the original singlet majoron model, though the decay mechanism is different, in our model nonvanishing contribution to this decay occurs at two loop level. To see what kind role the $\tau$ neutrino can play in the cosmology, we need to be more specific. We take $M_{R}=50 \mathrm{GeV}$ and $m_{\nu_{\tau}}=30 \mathrm{KeV}$ and find the life time $\tau \simeq 0.2 y \mathrm{r}$. A neutrino with this mass and life time can just be a late decaying particle which is required for the large scale structure formation of the universe in the cold dark matter model [18]. Nevertheless at the same time we have to require the $\nu_{\mu}$ to be lighter than a few $\mathrm{eV}$ in order not to violate the same requirement. The mass hierarchy between $\nu_{\tau}$ and $\nu_{\mu}$ is about one order of magnitude larger than that expected from above mentioned seesaw relation $m_{\tau}^{2} / m_{\mu}^{2}$, though we think it is still reasonable. Here again we see the advantage of the radiative seesaw model. Even the mass of $\nu_{\tau}$ is as small as $10 \mathrm{KeV}$, $M_{R}$ can be around $100 \mathrm{GeV}$ duo to the loop factor. So that $\nu_{\tau}$ can decay fast.

In conclusion, we discussed an new version of seesaw mechanism which is realized radiatively and gave a concrete model to exhibit its interesting new features. Generally speaking, in this mechanism the constrains from cosmology and astrophysics are relaxed compared with that in the tree-level seesaw model. we emphasize and focus on the weak scale seesaw in our model. The most interesting application of this new mechanism is on cosmology and neutrino physics. We pointed out that the lightest right-handed neutrino $\nu_{R}$ can be good candidate of cold dark matter. And at the same time light neutrino may provide a hot dark matter or late decaying particle for large scale structure formation, or offer solutions 
to other problems in neutrino physics. Finally we would like to point out that if $\nu_{R}$ is the dark matter of the universe, there are two possible ways to find out its signal. The first is through high energy collider experiment. $\nu_{R}$ pair can be produced through process like $e^{+} e^{-} \rightarrow \bar{\nu}_{R} \nu_{R}$. Since $\nu_{R}$ is invisible and a Majorana particle, the best signal is to search like sign charged lepton pair. Another way is to look for the annihilation products $\mu^{+} \mu^{-}$of dark matter $\nu_{R}$ pair in the indirect dark matter search experiments.

This work is supported by the National Science Foundation of China (NSFC).

\section{References}

[1] T. Yanagida, in Proceedings of Workshop on the Unified Theory and the Baryon Number in the Universe, Tsukuba, Japan, 1979, edited by A. Sawada and A. Sugamoto (KEK Report No. 79-188, Tsukuba, 1979); M. Gell-Mann, P. Ramond, and R. Slansky, in Supergravity, Proceedings of the Workshop, Stony Brook, New York, 1979, edited by D. Freedman and P. van Nieuwenhuizen (North-Holland, Amsterdam, 1980).

[2] G. Gyuk and M. S. Turner, Nucl. Phys. B (Proc. Suppl.) 38, 13 (1995); M. Kawasaki et al, Nucl. Phys. B419, 105(1994); S. L. Glashow, Phys. Lett. B 187, 367 (1987).

[3] For a review on solar and atmospheric neutrino problems, see A. Yu Smirnov, Talk given at 16th International Symposium on Lepton an Photon Interactions, Ithjaca, NY, 10-15 Aug. 1993.

[4] Q. Shafi and F.W. Stecker, Phys. Rev. Lett. 53, 1292 (1984); M. Davis, F. Summers, and D. Schlegel, Nature (London ) 359, 393 (1992); A. van Dalen and R. K. Schaefer, Astrophys. J. 393, 33 (1992); A. A. Klypin et al., ibid. 416, 1 (1993).

[5] S. Dodelson, G. Gyuk, and M. S. Turner, Phys. Rev. Lett. 72, 3754 (1994). 
[6] H. B. Kim and J. E. Kim; Nucl. Phys. B 433, 421 (1995).

[7] G. Blumenthal et al., Nature (London) 311, 517 (1984); J. P. Ostriker, Ann. Rev. Astron. Astrophys., 31, 689 (1993).

[8] V. Kuzmin, V. Rubakov and M. E. Shaposhnikov, Phys. Lett. B155, 16 (1985).

[9] M. Fukugita and T. Yanagida, Phys. Rev. D 42, 1295 (1990); J. Harvey and M. S. Turner, ibid., 3344 (1990).

[10] For a review, see R. Peccei, in Proceeding of the XXVI International Conference on High Energy Physics, Dallas, Texas, 1992; also see J. Cline, K. Kainulainen and K. Olive, Phys. Rev. Lett. 71, 2372 (1993); Phys. Rev. D 49, 6394 (1994).

[11] N. Turok and J. Zadrozny, Nucl. Phys. B 369, 729 (1992).

[12] The concept of radiative seesaw mechanism was first proposed by Babu and Mathur some years ago, see K. S. Babu and V. S. Mathur, Phys. Rev. D38, 3550 (1988). In their paper they discussed a left-right symmetric model. However in this work we propose a different model to realize this mechanism and therefore some very interesting and general features, showing up in our model, for the radiative seesaw mechanism were not noticed by Babu and Mathur.

[13] Y. Chikashige, R. Mohapatra and R. Peccei, Phys. Lett. B 98, 265 (1981); Phys. Rev. Lett. 45, 1926 (1980).

[14] D. Caldwell et al., Phys. Rev. Lett. 61 (1988) 510.

[15] For a review, see The Early Universe, eds E. W. Kolb and M. S. Turner (AddisonWesley Publishing Company, 1990).

[16] P. Chardonnet, P. Fayet and P. Salati, Nucl. Phys. B 394, 35 (1993). 
[17] LSND Collaboration, Phys. Rev. Lett. 75, 2650 (1995); J. E. Hill, ibid. 75, 2654 (1993).

[18] see equation (14) of reference 6.

[19] T. Walker et al., Astrophys. J. 376, 51 (1991).

[20] M. Morgan and G. Miller, Phys. Lett. B 179, 379 (1986). 